\title{
Aflatoxinas y tricotecenos en trigo y derivados: incidencia de la contaminación y métodos de determinación
}

\author{
Trichothecenes and aflatoxins contamination \\ in wheat and wheat products: occurrence \\ and methods of determination
}

\begin{abstract}
Trichothecenes and aflatoxins are natural contaminants produced by filamentous fungi and they are widely present in wheat. The presence of these mycotoxins are a serious public health problem because they remains stable during the industrial processes to which wheat is subjected when manufacturing derived products and due the toxic effects that can cause in human health. The aim of this review was to address the main topics related with the occurrence and quantification methods commonly used for determination of tricothecens (deoxynivalenol, nivalenol, T2-toxin and HT-2 toxin) and aflatoxins in wheat and wheat products. It can be observed that, in the last years, there was an increase in the number of papers published in international journals containing these subjects. Many studies have reported levels above the maximum allowable limits and the presence of aflatoxins and trichothecenes have also been associated with the simultaneous occurrence of other mycotoxins. The best way to reduce aflatoxin and tricothecenes contamination in wheat and wheat products is still the application of good agricultural practices for growing, harvesting and storage of grains, because although there are methods for decontamination, these are not yet implemented on a wide scale.

Key words: Mycotoxins, DON, analytical methods, HPLC, fusarium spp, aspergillus spp.
\end{abstract}

\section{INTRODUCCIÓN}

Las micotoxinas son definidas en general como metabolitos secundarios tóxicos de bajo peso molecular, producidos por varias especies de hongos filamentosos en sustratos donde estos se estén desarrollando en condiciones ideales de humedad y temperatura (1).

Son considerados como uno de los más importantes contaminantes naturales en los alimentos, debido a la alta incidencia en productos de origen vegetal como los cereales y especias, así como en productos cárnicos y lácteos (1-4).

La contaminación por micotoxinas puede ocurrir tanto en la fase agrícola como durante el almacenamiento o procesamiento de los alimentos y representa un problema de salud pública debido a la alta toxicidad de estas sustancias en la salud humana y animal, y también porque son moléculas altamente estables a los procesos industriales a los cuales se someten los alimentos (1, 4-6).
Felipe M. Trombete (1) Tatiana Saldanha (2) Glória M. Direito (3) Marcelo E. Fraga (4)

1,2 Departamento de Tecnologia de Alimentos (DTA), Universidade Federal Rural do Rio de Janeiro/UFRRJ, Brasil 3,4Departamento de Microbiologia e Imunologia Veterinária (DMIV) - Universidade Federal Rural do Rio de Janeiro/UFRRJ, Brasil

Dirigir la correspondencia a: Señor

Felipe M. Trombete

Departamento de Tecnología de Alimentos PPGCTA, Rodovia BR 465, km 7, UFRRJ Seropédica-RJ, 23890-000, Brasil.

E-mail: trombete@ufrrj.br

Este trabajo fue recibido el 11 de Diciembre de 2012 y aceptado para ser publicado el 10 de Marzo de 2013.

Actualmente, se conocen más de 300 micotoxinas, sin embargo, cerca del 30 son consideradas de mayor importancia debida a la alta toxicidad y amplia presencia en los alimentos (7).

En los cereales, la presencia de hongos de los géneros Aspergillus, Penicillium y Fusarium están directamente relacionados con la contaminación de los alimentos por micotoxinas, además de causar enfermedades importantes de los cultivos $(8,9)$.

Las principales micotoxinas producidas por diferentes especies de hongos y de gran ocurrencia en los cereales son los tricotecenos (principalmente deoxinivalenol - DON, nivalenol - NIV, toxina T-2 y HT-2), zearalenona (ZEA), ocratoxina A (OTA), fumonisinas y las aflatoxinas del grupo B y $G$ (10-17).

En el trigo y otros cereales de invierno, el hongo Fusarium graminearum (fase sexual: Gibberella zeae) es considerado un importante patógeno que causa la enfermedad denomi- 
nada Fusarium Head Blight (fusariosis) que además de causar pérdidas en la calidad del grano también promueve la contaminación del cultivo por tricotecenos, ZEA y otras Fusarium toxinas (18-22).

En general, la ingestión de alimentos contaminados con tricotecenos (TCTs), como DON, NIV, T-2 y HT-2, provoca en los animales la inhibición de la síntesis de proteínas, el daño celular y consiguiente inmunosupresión, inhibición del apetito, vómitos, diarrea, dermatitis y sangrando, con síntomas similares en los seres humanos (23-25).

Del grupo de los TCTs, DON es la micotoxina más frecuente de la dieta humana $(24,26)$. La Agencia Internacional de Investigación sobre el Cáncer (IARC) no clasifica los TCTs como cancerígenos para los humanos (3), pero de acuerdo con la Organización Mundial de la Salud (WHO), la presencia de DON es un problema de salud pública y los síntomas de intoxicación aguda, tales como vómitos dentro de treinta minutos, puede confundirse con la intoxicación causada por Bacillus cereus (27).

En países de climas más elevados, la contaminación por aflatoxinas (AFs) producidas por Aspergillus (especialmente A. flavus y A. parasiticus) también han sido frecuentemente identificada en altos niveles en el trigo y en los derivados (8, 28, 29).

Las aflatoxinas son consideradas agentes cancerígenos naturales con mayor potencial tóxico para el hombre (30) y es reconocido por la IARC como agentes hepatotóxicos e cancerígenos para los humanos (grupo 1A), capaz de inducir cáncer de hígado, cirrosis hepática y reducir la resistencia inmune, causando brotes de hepatitis viral tipo B (3).

En 2011, el informe presentado por la Comisión Europea (CE) a través del sistema RASFF (Rapid Alert System for Food and Feed) tuvo 585 casos de alimentos contaminados con aflatoxinas y 11 notificaciones para DON. De este total, 24 de ellos estaban relacionadas con harina de trigo y otros productos de panificación (4).

La contaminación por TCTs y aflatoxinas en trigo es considerada una preocupación constante en muchos países debido a la alta ocurrencia y a los altos índices encontrados tanto en materia prima como en derivados. El objetivo de este estudio fue realizar una revisión de la literatura abordando las cuestiones principales relacionados con la ocurrencia y los métodos de determinación de los tricotecenos (DON, NIV, T-2 y HT-2) y las aflatoxinas en el trigo y derivados a través de los estudios publicados en revistas internacionales en los últimos años.

\section{LIMITES MAXIMOS PERMISIBLES DE AFS Y TCTS EN EL TRIGO Y DERIVADOS}

De acuerdo con la Organización de las Naciones Unidas para la Agricultura y la Alimentación - FAO (31), establecer normas para las micotoxinas es una actividad compleja que envuelve muchos factores. Estos incluyen información sobre los efectos en la salud humana y animal, niveles de exposición humana, factores comerciales y hasta la disponibilidad de métodos de muestreo y análisis eficiente.

En lo que respecta a la contaminación por micotoxinas en trigo y sus derivados, la presencia de estas sustancias debe seguir el principio ALARA (As Low As Reasonably Achievable), presente tan bajo como sea razonablemente posible (1).

Los límites relativos a las aflatoxinas se refieren generalmente a la presencia de aflatoxina B1 (AFB1) y/o las aflatoxinas totales, siendo esta última la suma de los índices de $A F B_{1}$, $A \mathrm{FB}_{2}, \mathrm{AFG}_{1}$ y $\mathrm{AFG}_{2}$. El máximo valor aceptable de los países de los niveles de aflatoxinas en el trigo para el consumo humano, corresponde a 5 ppb (partes por billón). El valor más alto aceptable se establece para la India que corresponde a $30 \mathrm{ppb}$ (32).

En cuanto a TCTs, los límites normativos son dirigidos a la presencia de DON, NIV y la toxina HT-2 y solamente Rusia estipula niveles máximos para toxina T-2. Según la $C E$, la legislación para DON es suficiente para proteger a los consumidores de la exposición a otras toxinas de Fusarium spp, dada la ocurrencia simultánea de estos compuestos (24).

El índice máximo aceptable para DON varía de 750 ppb en la Unión Europea (UE) (24), a 2000 ppb en Canadá y Brasil $(33,34)$. Por lo general, los límites más estrictos son establecidos por la CE que se aplica en los 27 estados miembros. Sin embargo, incluso en los países de la UE existe una discrepancia en los valores máximos para las micotoxinas. En España, el límite máximo para AFs es de 10 ppb y en Austria este valor corresponde a $1 \mathrm{ppb}(35,36)$.

\section{TABLA 1}

Límites máximos establecidos por distintos países para las aflatoxinas totales y deoxinivalenol en granos de trigo para el consumo humano.

\begin{tabular}{|c|c|c|c|}
\hline Micotoxina & País & Máximo aceptado (ppb) & Referencia \\
\hline Aflatoxinas Totales & Unión Europea & 4 & (37) \\
\hline (Suma de $\mathrm{AFB}_{1}$ & Turquía & 4 & (38) \\
\hline \multirow[t]{6}{*}{$\mathrm{AFB}_{2}, \mathrm{AFG}_{1}, \mathrm{AFG}_{2}$ ) } & Chile & 5 & (5) \\
\hline & Brasil & 5 & (34) \\
\hline & India & 30 & (36) \\
\hline & Rusia & 700 & (36) \\
\hline & Unión Europea & 750 & (35) \\
\hline & USA & 1000 & (39) \\
\hline \multirow[t]{4}{*}{ Deoxinivalenol } & China & 1000 & $(40)$ \\
\hline & Japón & 1100 & (36) \\
\hline & Canadá & 2000 & (33) \\
\hline & Brasil & 2000 & (34) \\
\hline
\end{tabular}


En la tabla 1 están resumidos los límites máximos para la presencia de aflatoxinas y DON en granos del trigo, determinado por los diferentes países. Cabe señalar que en algunos como USA, Canadá y UE también se establecen límites específicos y más estrictos para los productos destinados al consumo para lactantes y niños.

Tal como se observa en la tabla 1, los niveles para la presencia de estas toxinas se encuentran en partes por mil millones. Por lo tanto, para evaluar la ocurrencia de estas toxinas son necesarias metodologías de identificación que sean precisas, exactas, sensibles y al mismo tiempo, rápidas y convenientes.

\section{MÉTODOS PARA DETERMINAR AFs Y TCTS Extracción}

La AFS y los TCTs son moléculas solubles en disolventes polares tales como metanol, acetonitrilo y agua. Por lo tanto, estos son los más utilizados para la extracción en granos y productos de trigo. La figura 1 contiene ilustraciones de las estructuras moleculares de los principales TCTs y AFs.

Las proporciones de los disolventes utilizados en las soluciones de extracción de estas micotoxinas son variables de acuerdo con la muestra. Así, los autores optimizan la composición de la solución de acuerdo con los mejores resultados de recuperación obtenidos. En la tabla 2 están detalladas las

\section{FIGURA 1}

Estructuras moleculares de las aflatoxinas y tricotecenos.<smiles>COc1cc2c(c3oc(=O)c4c(c13)CCC4=O)C1C=COC1O2</smiles>

E

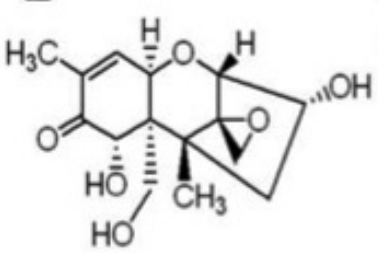<smiles>COc1cc2c(c3oc(=O)c4c(c13)CCC4=O)C1CCOC1O2</smiles>

F

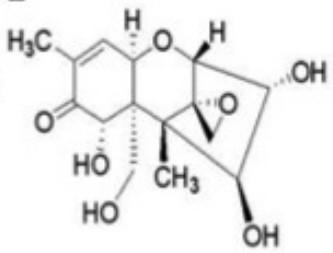<smiles>COc1cc2c(c3oc(=O)c4c(c13)C1C=COC4OC1)C(=O)OC2</smiles>

G

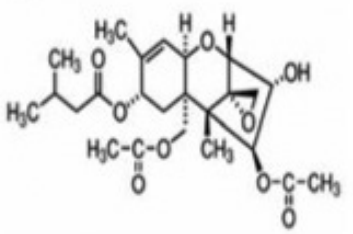<smiles></smiles>

H

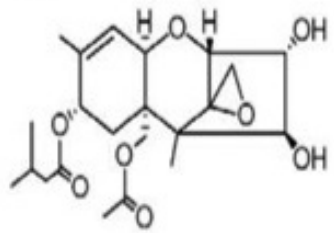

A - Aflatoxina $B_{1} ; B$ - Aflatoxina $B_{2} ; C$ - Aflatoxina $G_{1} ; D$ - Aflatoxina $G_{2}-E$ - Deoxinivalenol; F- Nivalenol; $G$ - Toxina T-2; H - Toxina HT-2. Fuente: European Comission (41),

TABLA 2

Soluciones de extracción utilizadas en investigaciones de aflatoxinas y tricotecenos en el trigo y productos derivados.

\begin{tabular}{|c|c|c|c|c|}
\hline \multirow[t]{5}{*}{ Micotoxina } & Matriz & Disolvente de extracción & Proporción & Referencia \\
\hline & Granos, & & 80: 20 & $(42,43,44,45)$ \\
\hline & harina y derivados & metanol: agua & 70: 30 & (8) \\
\hline & & & 50: 50 & $(20)$ \\
\hline & & acetona: agua & 84: 16 & (46) \\
\hline \multirow[t]{4}{*}{ Aflatoxinas } & Granos & acetona: agua (ph 1,5) & 70: 30 & (47) \\
\hline & & acetonitrilo: sol. $\mathrm{NaCl} 4 \%$ & 90: 10 & (29) \\
\hline & & a pH 1,5 & & (48) \\
\hline & Granos y harina & agua + polietilenglicol & 20: 1 & $(42,49)$ \\
\hline \multirow[t]{4}{*}{ Deoxinivalenol } & Granos & metanol: agua & 50: 50 & (20) \\
\hline & & agua & 1 & (50) \\
\hline & Granos y & & 84: 16 & $(51,52)$ \\
\hline & harina integral & acetonitrilo:agua & 70: 30 & $(53)$ \\
\hline \multirow[t]{3}{*}{ T-2 e HT-2 } & Granos & metanol: agua $+\mathrm{NaCl}$ & 90: 10 & (54) \\
\hline & & & 70: 30 & (50) \\
\hline & & metanol: agua & 70: 20 & (20) \\
\hline
\end{tabular}


diferentes composiciones de las soluciones de extracción utilizadas en la determinación de las aflatoxinas y DON en el trigo.

En general, en las investigaciones de TCTs, son utilizadas una única solución capaz de extraer todas las sustancias de interés, tales como DON, NIV, T-2 y HT-2. Del mismo modo, en determinación de multimicotoxinas son optimizadas las proporciones de los disolventes a las que en un solo paso de extracción sea posible la solubilización de diversas micotoxinas. Skrbic et al (55) propusieron el uso de una solución de acetonitrilo: agua (84: 16, v/v) para la extracción de AFs, OTA, ZEA, DON, T 2-, HT-2 y fumonisinas.

Sulyok et al (56) evaluaron una solución de extracto para 39 micotoxinas en trigo y maíz utilizando acetonitrilo: agua: ácido acético (79: 20: 1, v/v/v), consiguiendo buenos resultados analíticos.

Durante el proceso de extracción, el uso de columnas de inmunoafinidad (Immunoaffinity Column - IAC) han sido ampliamente utilizadas para purificación del extracto y concentración de las micotoxinas por anticuerpos específicos que interactúan con la toxina en los alimentos. Así se eliminan los interferentes y la evaluación se hace más fiable $(9,45,57,58)$.

De acuerdo con Ramos et al (59), la metodología para cuantificación de las aflatoxinas que utiliza la purificación por IAC tiene la desventaja de ser seis veces más cara que el método que no la utiliza, por lo tanto, se debe evaluar la necesidad de la utilización de este método durante la etapa de extracción de las micotoxinas.

\section{Cuantificación}

Para cuantificar las AFs y TCTs en trigo se han desarrollado, optimizado y validado diferentes metodologías. En la mayoría de las técnicas de estudios son utilizadas Cromatografía Líquida de Alta Resolución (High Performance Liquid Chromatography - HPLC), Cromatografía Líquida de Ultra Rendimento (Ultra Performance Liquid Chromatography - UPLC) o Cromatografía de Capa Fina (Thin Layer Chromatography - TLC).

Por ser fluorescente cuando son expuestas a la luz UV, en las dos primeras técnicas, la detección se realiza con frecuencia por fluorescencia (Fluorescence Detector - FLD), ultravioleta (Ultraviolet Detector - UV) o bien por espectrómetro de masas (Mass Spectrometry - MS) $(29,53,60)$.
Para Fujii et al (61) las metodologías por TLC tienen la ventaja de ser simples y de bajo costo, con la visualización directa de los perfiles cromatograficos basados en el color, fluorescencia y aspectos de la corrida. Por lo tanto, es una técnica que se ha utilizado en estudios recientes para determinar AFs en el trigo $(46,62,63)$.

HPLC es la técnica más utilizada para cuantificación de estas micotoxinas. La columna que se utiliza con mayor frecuencia es la de fase inversa (C18), y la fase móvil varía mucho entre los trabajos. En la tabla 3 están detalladas la composición de diversas fases móviles utilizadas para estudios de cuantificación de AFs y DON mediante HPLC-FLD o HPLC-UV.

Otros métodos basados en reacciones inmunoquímicas y detecciones rápidas Ilamadas "ensayo de inmunoabsorción enzimática" o ELISA (Enzyme-linked immunosorbent assay), también se han utilizado para este propósito. Son económicos y proporcionan resultados precisos y reproducibles, capaces de identificar la presencia de bajos niveles de micotoxinas en ppm o ppb, por lo general con la obtención de respuesta visual de presencia o ausencia $(56,65)$.

Por lo tanto, se puede utilizar como métodos de cuantificación directo o como procedimientos de detección rápido, debido a la facilidad de manipulación $(8,28,66)$.

Sin embargo, los métodos por ELISA tienen la desventaja de presentar posibles resultados falsos positivos y por lo tanto puede requerir una confirmación adicional del resultado (67). La CE recomienda la confirmación de resultados positivos por HPLC-FLD (68).

Nuevas metodologías tales como la microextracción líquido-líquido dispersiva (7) y el uso de isótopos estables como trazadores $(57,69)$ también son una opción para la cuantificación de las AFs e TCTs en el trigo y derivados.

Entre las metodologías existentes, la elección dependerá de factores económicos, el propósito del análisis, el equipo de analistas, entre otros. Es fundamental utilizar métodos confiables y por lo tanto la validación constante es esencial en las investigaciones de AFs e TCTs en trigo.

En la actualidad, el uso de HPLC y MS son los métodos para cuantificar aflatoxinas y tricotecenos más utilizados y adoptados como oficiales por los países productores o importadores de trigo y derivados.

\section{TABLA 3}

Composición de fases móviles utilizadas en la detección de aflatoxinas y deoxinivalenol mediante HPLC con columna $\mathrm{C}_{18}$ y detección por fluorescencia o ultravioleta.

Micotoxina

Fase Móvil

agua: acetonitrilo: ac. acético

Aflatoxinas

agua: metanol: acetonitrilo

agua: acetonitrilo

Deoxinivalenol
Proporción (volumen)

99: $99: 2$

65: 20: 15

55: $45: 2$

64: 18: 18

62: $22: 16$

60: $20: 20$

96: $2: 2 \rightarrow 0: 0: 1$

93: 7

85: 15

85: $15 \rightarrow 78: 22$

88: 12
Referencia

agua: metanol 


\section{OCURRENCIA DE AFs E TCTS \\ EN TRIGO Y DERIVADOS}

Debido a la alta incidencia de hongos del género Aspergillus, Fusarium y Penicillium en trigo, cuando se recoge y se almacena en condiciones de altas temperaturas y humedad, presencia de insectos, daños mecánicos a los granos y transporte inadecuado, es posible observar el aumento de los niveles de contaminación por micotoxinas y por consiguiente de los productos derivados $(12,13,67,70)$.

La preocupación por la contaminación del trigo por micotoxinas, se ha intensificado en los últimos años, como puede verse por el aumento del número de publicaciones sobre la materia. Anualmente son publicados cerca de 160 trabajos científicos en revistas internacionales relacionados con deoxynivalenol y trigo. Por los términos "aflatoxina" y "trigo" se publican aproximadamente 30 artículos anualmente, como puede verse en la figura 2 .

En la literatura científica, varios estudios han buscado cuantificar los niveles de contaminación por AFs y TCTs en el trigo y sus productos derivados en los diferentes países. En estos trabajos se puede observar que estas micotoxinas están ampliamente distribuidas y algunas veces en niveles superiores a los límites máximos permisibles. En la tabla 4 están resumidos estos estudios.

En general, la contaminación por micotoxinas ocurre de forma simultánea y no apenas por sólo un grupo aislado. Ghali et al (28) y Terken et al (73) relacionaron la presencia de aflatoxinas con la presencia de OTA en granos del trigo y harinas. Muthomi et al (20) asocia la presencia de DON, T-2 y ZEA en granos del trigo comercializado en Kenia. Estos datos son alarmantes, ya que no se conoce bien los posibles efectos sinérgicos asociados con la ingesta de diferentes micotoxinas en la salud humana.

Para la reducción y descontaminación de micotoxinas en los productos destinados al consumo humano, se han desarro- llado y adaptado distintas metodologías basadas en procesos físicos, químicos y microbiológicos. Entre estos se puede mencionar el uso de agentes químicos, biotransformadores, adsorbentes, métodos basados en la clasificación por color, radiación ionizante, ozonización y varios otros (7).

Sin embargo, a pesar de los métodos citados que se consideran prometedores, el procedimiento principal para el control de la contaminación del trigo con las micotoxinas todavía es el uso de las Buenas Practicas Agrícolas en toda la cadena prodctiva, ya que, cuando se forman las micotoxinas, estas no serán eliminadas fácilmente durante el procesamiento de los alimentos.

\section{COMENTARIOS FINALES}

A partir de esta revisión, puede observarse que la incidencia de aflatoxinas y tricotecenos en los granos de trigo para consumo humano han sido el objetivo de varios estudios recientes en el área micotoxicológica y es considerado un problema de salud pública. Los niveles encontrados en muchos trabajos son superiores a los límites reglamentarios para la presencia de estas toxinas los que varían mucho entre los países. Para la evaluación y supervisión de esta contaminación, es necesario el uso de métodos analíticos fiables. La cromatografía líquida de alta resolución, la cromatografía en capa fina y kits de detección rápida basadas en reacciones inmunoenzimáticas son las metodologías más utilizadas. Varias otras técnicas también se han adaptado, optimizado y validado para este propósito. Procedimientos alternativos, utilizando nuevas tecnologías para la descontaminación de las aflatoxinas y tricotecenos en el trigo y otros alimentos han sido estudiados, pero hay todavía muchos factores que restringen su aplicabilidad práctica. Por lo tanto, la adopción de buenas prácticas agrícolas en toda la cadena productiva del trigo sigue siendo la mejor manera de prevenir contaminación por tricotecenos y aflatoxinas.

\section{FIGURA 2}

Publicaciones en revistas científicas internacionales desde el año 2000 sobre trabajos con temas relacionados a las aflatoxinas e deoxinivalenol.

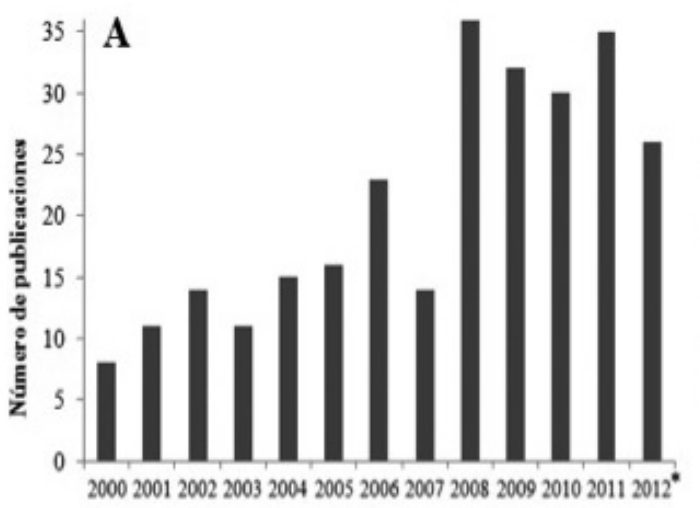

Año

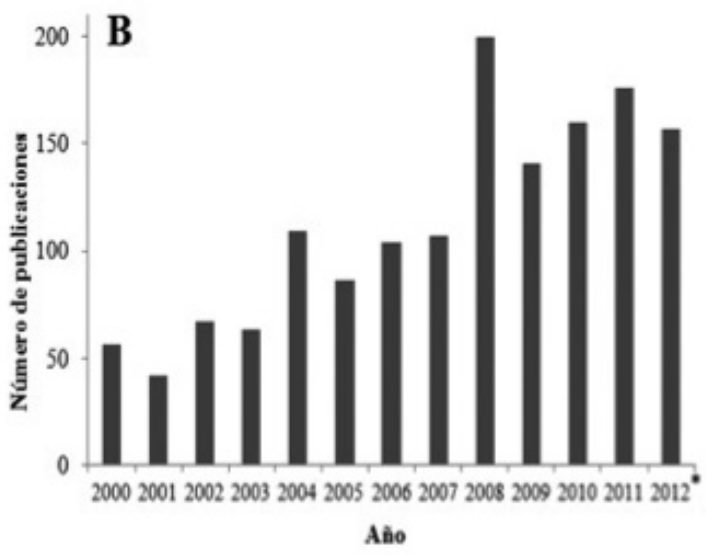

Ah้o

*2012: resultados parciales. A - Palabras clave: "aflatoxin" + "wheat"; B - Palabras clave: "deoxynivalenol" + "wheat". Fuente: Web of Knowledge (71). 


\section{RESUMEN}

Los tricotecenos y las aflatoxinas son contaminantes naturales producidos por hongos filamentosos y de alta incidencia en trigo. La presencia de estas micotoxinas es un problema importante de salud pública debido a que son moléculas muy estables durante los procesos de industrialización a los que el trigo es sometido para la obtención de derivados y por los efectos tóxicos que causan. El objetivo de esta revisión fue abordar los principales temas relacionados con la incidencia y lós métodos de determinación de los tricotecenos (deoxinivalenol, nivalenol, toxina T-2 y HT-2) y las aflatoxinas en el trigo y derivados. Se observa que, en los últimos años, ha aumentado el número de trabajos publicados en revistas internacionales sobre el tema. En muchos estudios se demuestra niveles superiores a los límites máximos permitidos y la presencia de aflatoxinas y tricotecenos también se han asociado con la ocurrencia simultánea de otras micotoxinas. La mejor manera de reducir la contaminación de trigo por tricotecenos y aflatoxinas sigue siendo la aplicación de buenas prácticas agrícolas para el cultivo, cosecha y almacenamiento de granos, ya que, aunque hay métodos de descontaminación, estos aún no son aplicados a gran escala.

Palabras clave: Micotoxinas, DON, métodos analíticos, HPLC, fusarium spp, aspergillus spp.

\section{BIBLIOGRAFÍA}

1. EFSA - European Food Safety Authority. Mycotoxins. 2012. Disponible en: http://www.efsa.europa.eu/en/aboutefsa. htm. Consultado en Octubre de 2011.

2. Chile. Ministerio de Salud. Reglamento Sanitario de los Alimentos. Art. 169, decreto 977/96, del Ministerio de Salud, 1996.

3. IARC. International Agency for Research on Cancer. Mono- graph on the evaluation of carcinogenic risk to humans, World Health Organization, some traditional herbal medicines, some mycotoxins, naphthalene and styrene. Summary of data reported and evaluation. Lyon, 2002; 82: 171-5.

4. RASFF. The Rapid Alert System for Food and Feed. Annual Report. EU, Luxembourg, 2012. Disponible en: http:// ec.europa.eu/RASFF. Consultado en Octubre de 2012.

5. Chile. Ministerio de Salud. Departamento de Alimentos y Nutrición. Antecedentes generales sobre las aflatoxinas y otras micotoxinas y elementos a tener en cuenta para el diseño de prácticas correctas de cultivo y elaboración de nueces, 2007;1:36.

6. Kabak B. The fate of mycotoxins during thermal food processing. J Sci Food Agr 2009; 89: 549-54.

7. EFSA. European Food Safety Authority. Review of mycotoxin-detoxifying agents used as feed additives: mode of action, efficacy and feed/food safety. Scientific Report CFP/EFSA/FEEDAP/2009/01, 2009.

8. Aydin A, Gunsen U, Demirel S. Total aflatoxin, aflatoxin $B 1$ and ochratoxin A levels in Turkish wheat flour. J Food Drug Anal 2008; 16: 48-53.

9. Zaied C, Zouaoui N, Bacha H, Abid S. Natural occurrence of citrinin in Tunisian wheat grains. Food Control 2012; 28: 106-9.

10. Cover JS, Teixeira ML, Souza LFO, Fuentefría AM. Determinación de zearalenona por cromatografía líquida de alta resolución: normalización de un nuevo método para raciones especiales para cerdos. Rev Chil Nutr 2010; 37:369-75.

11. FSA. Food Standards Agency. The UK Code of Good Agricultural Practice to Reduce Fusarium Mycotoxins in Cereals, 2007.

12. Juan C, Ritieni A, Manes J. Determination of trichothecenes and zearalenones in grain cereal, flour and bread by liquid

\section{TABLA 4}

Distribución de los tricotecenos y aflatoxinas en trigo y productos derivados.

\begin{tabular}{|c|c|c|c|c|c|c|c|c|}
\hline País & Micotox & Matriz & Técnica* & $\mathrm{n}$ & Positivas & Rango (ppb) & Promedio (ppb) & Referencia \\
\hline BRA & DON & Granos & HPLC-MS & 65 & $64(98 \%)$ & ND - 1250 & 540 & (53) \\
\hline SRB & DON & Granos & ELISA & 103 & 94 (91\%) & $50-3306$ & 444,18 & $(50)$ \\
\hline SRB & DON & Harina & UPLC-MS & 15 & $13(87 \%)$ & $17,5-976$ & 325 & (55) \\
\hline KEN & DON & Granos & ELISA & 82 & $56(68 \%)$ & $105-303$ & 122 & (20) \\
\hline BRA & DON & Granos & HPLC-MS & 164 & $80(48 \%)$ & ND-8310 & $290^{A P}$ & (72) \\
\hline BRA & DON & Salvado & HPLC-MS & 449 & $217(48 \%)$ & ND-3129 & $424^{\mathrm{AP}}$ & (72) \\
\hline RUS & DON & Granos & HPLC-FLD & 2166 & $1498(69 \%)$ & $50-8600$ & 0,4 & $(52)$ \\
\hline QAT & DON & Granos e Harina & HPLC-UV & 10 & $2(20 \%)$ & $148-183$ & - & (42) \\
\hline BRA & NIV & Granos & HPLC-MS & 65 & $54(83 \%)$ & ND - 781 & 337 & (53) \\
\hline SRB & $\mathrm{T}-2$ & Granos & ELISA & 103 & $66(64 \%)$ & $60-495$ & 129,13 & (50) \\
\hline KEN & $\mathrm{T}-2$ & Granos & ELISA & 80 & $61(76 \%)$ & $20-66$ & 26 & (20) \\
\hline LBN & AFs & Granos & HPLC-FLD & 156 & $119(76 \%)$ & ND-12,2 & - & (29) \\
\hline ESP & AFs & Granos & UPLC-FLD & 14 & 0 & - & - & $(47)$ \\
\hline TUR & AFs & Harina & ELISA & 100 & 45 (45\%) & $0,05-14$ & 0,79 & (8) \\
\hline TUN & AFs & Granos & ELISA & 51 & 15 (30\%) & $4-19,2$ & 6,7 & (28) \\
\hline TUR & AFs & Granos & HPLC-FLD & 41 & $24(59 \%)$ & $0,01-0,64$ & & $(42)$ \\
\hline KEN & $\mathrm{AFB}_{1}$ & Granos & ELISA & 41 & $23(56 \%)$ & $0-7$ & 1,9 & (20) \\
\hline
\end{tabular}

DON - Deoxinivalenol. AFs - Aflatoxinas totales. NIV - Nivalenol. * HPLC - Cromatografía Liquida de Alta Resolución. MS - Espectrometría de masas. ELISA - Ensayo de Inmunoabsorción Enzimática. PDA - Detector Arreglo de Diodo. FLD - Detector de fluorescencia. UV - Detector de Ultravioleta.

$\mathrm{n}=$ número de muestras analizadas. $\mathrm{AP}=$ Promedio sobre muestras positivas . 
chromatography tandem mass spectrometry. Food Chem 2012; 134: 2389-97.

13. Kachuei $R$, Yadegari1 $M H$, Rezaie $S$, Allameh A, Safaie $N$, Zaini $F$, Khanezad YF. Investigation of stored wheat mycoflora, reporting the Fusarium cf. langsethiae in three provinces of Iran during 2007. Ann Microbiol. 2009; 59: 383-90.

14. Villa P, Markaki P. Aflatoxin B1 and Ochratoxin A in breakfast cereals from Athens market: Occurrence and risk assessment. Food Control. 2009; 20: 455-61.

15. Pleadin J, Sokolovic $M$, Persi $N$, Zadravec $M$, Jak $V$, Vulic A. Contamination of maize with deoxynivalenol and zearalenone in Croatia. Food Control. 2012; 28: 94-8.

16. Roohi S, Azizi IG, Hashemi M. Fumonisin contamination based on flour quality used in bakeries and confectioneries in Qaemshahr (city of the Northern Iran). Afr J Microbiol Res. 2012; 6: 1815-8.

17. Roscoe $V$, Lombaert GA, Huzel V, Neumann G, Melietio J, Kitchen D, Kotello S, Krakalovich T, Trelka R, Scott PM. Mycotoxins in breakfast cereals from the Canadian retail market: a 3-year survey. Food Addit Contam Part A. 2008; 25: 347-55

18. Infantino $A$, Santori $A$, Shah DA. Community structure of the Fusarium complex on wheat seed in Italy. Eur J Plant Pathol. 2012, 132: 499-510.

19. Koch HJ, Pringas C, Maerlaender B. Evaluation of environmental and management effects on Fusarium head blight infection and deoxynivalenol concentration in the grain of winter wheat. Eur J Agron. 2006; 24: 357-66.

20. Muthomi JW, Ndung'u KK, Gathumbi JK, Mutitu EW, Wagacha JM. The occurrence of Fusarium species and mycotoxins in Kenyan wheat. Crop Protection. 2008; 27: 1215-1219.

21. Son $H$, Lee YW. Fusarium graminearum mycotoxins and their biosynthetic genes. Mycotoxins 2012; 62: 29-40.

22. Teixeira $M L$, Pertuzzatti $D$, Leite $D C$, Oliveira LF, Fuentefria AM. Determinación de ocratoxina-A por HPLC con detección por fluorescencia (HPLC-FL): un nuevo método estandarizado para muestras de trigo. Rev Chil Nutr. 2010; 37:184-91.

23. Dilkin P. Micotoxicose suína: aspectos preventivos, clínicos e patológicos. Biológico 2002; 64: 187-91.

24. EC. European Commission. Health e Consumer protection directorate general. Opinion of the Scientific Committee on Food on Fusarium toxins. Part 6: Group evaluation of T-2 toxin, HT-2 toxin, nivalenol and deoxynivalenol SCF/ CS/CNTM/MYC/27 Final. 2002a.

25. Minervini F, Fornelli F, Flynn KM. Toxicity and apoptosis induced by the mycotoxins nivalenol, deoxynivalenol and fumonisin B1 in a human erythroleukemia cell line. Toxicol In Vitro 2004; 18: 21-8.

26. Wartha $B$, Sulyoka M, Fruhmannb P, Berthillera F, Schuhmachera R, Hametnerb C, Adamc G, Fröhlichb J, Krskaa $R$. Assessment of human deoxynivalenol exposure using an LC-MS/MS based biomarker method. Toxicol Lett. 2012; 211: 85-90.

27. WHO. World Health Organization. Global Strategy for Food Safety: safer food for better health. Food Safety Programme. Geneva, 2002.

28. Ghali R, Hmaissia-khlifa $K$, Ghorbel H, Maaroufi $K$, Hedili A. Incidence of aflatoxins, ochratoxin $A$ and zearalenone in tunisian foods. Food Control 2008; 19: 921-24.

29. Joubrane K, Khoury AEL, Lteif R, Rizk T, Kallassy M, Hilan $C$, Maroun R. Occurrence of aflatoxin $B 1$ and ochratoxin
A in Lebanese cultivated wheat. Mycotoxin Res. 2011; 27: 249-57.

30. Songsermsakul P, Razzazi-Fazeli E. A review of recent trends in applications of liquid chromatography-mass spectrometry for determination of mycotoxins. J Liq Chromatogr $R$ T. 2008; 31: 1641-86.

31. FAO. Food and Agriculture Organization of the United Nations. Worldwide regulations for mycotoxins in food and feed in 2003. Hans P, Egmond V, Jonker MA. Rome, 2004.

32. India. Ministry of health and family welfare. Food Safety and Standards Authority of India. Notification. Food Safety and Standard (Contaminants, Toxins and Residues) Regulation, New Delhi, 2011.

33. Canada. Health Canada. Canadian Standards (Maximum Levels) for Various Chemical Contaminants in Foods. 2012.

34. Brasil. Ministério da Saúde. Agência Nacional de Vigilância Sanitária. Resolução de Diretoria Colegiada n.7, de 18 de fevereiro de 2011. Limites máximos tolerados para micotoxinas em alimentos. Diário Oficial da República Federativa do Brasil. Brasília, 2011.

35. EC. European Commission. Comission Regulation $N^{\circ}$ 401/2006 of 23 February 2006. Laying down the methods of sampling and analysis for the official control of the levels of mycotoxins in foodstuffs. Official J European Union 2006; 12-34.

36. EMAN - European Mycotoxins Awareness Network. Mycotoxins Legislation Worldwide. 2012. Disponible en: http://services.leatherheadfood.com/eman/FactSheet. aspx?ID=79. Consultado en Octubre de 2011.

37. EC. European Comission. Commission Regulation n.165/2010 of 26 February 2010 amending Regulation EC n. 1881/2006 setting maximum levels for certain contaminants in foodstuffs as regards aflatoxins. Official J European Union 2010.

38. Turkish Food Codex. Gida maddelerinde belirli bulaoanlaran maksimum seviyelerinin belirlenmesi hakkinda teblig. Resmi Gazete 2002; Ankara: Basbakanlik Basimevi.

39. FDA - Food and Drug Administration. Advisory for deoxynivalenol (DON) in finished wheat products intended for human consumption and in grain and grain by-products for animal Feed, 2010.

40. China. Ministry of Health. National Food Safety Standards. GB 2761-2011. Maximum levels of mycotoxins in foods, 2011.

41. EC. European Comission. Joint Research Centre. Institute for reference material and measurements. LERDA, D. Mycotoxins Factsheet. 4 ed. JRC Technical Notes 2011.

42. Abdulkadar AHW, Al-Ali AA, Al-Kildi AM, Al-Jedah JH. Mycotoxins in food products available in Qatar. Food Control 2003; 15: 543-8.

43. Campone L, Piccinelli AL, Celano R, Rastrelli L. Application of dispersive liquid-liquid microextraction for the determination of aflatoxins B1, B2, G1 and $G 2$ in cereal products. J Chromatogr A 2011; 1218: 7648-54.

44. Gallo G, Bianco ML, Bognanni R, Saimbene G. Mycotoxins in durum wheat grain: Hygienic-health quality of sicilian production toxicology and chemical. J Food Sci. 2008; 73: 42-7.

45. Giray B, Girgin G, Enginn AB, Aydin S, Sahin G. Aflatoxin levels in wheat samples consumed in some regions of Turkey. Food Control 2007; 18: 23-9.

46. Saleemullah Al, Iqtidar AK, Hamidullah S. Aflatoxin contents of stored and artificially inoculated cereals and nuts. Food Chem. 2006; 98: 699-703. 
47. Ibáñez-Vea M, Martínez R, Gonzáles-Peñas E. Cooccurrence of aflatoxins, ochratoxin $A$ and zearalenone in breakfast cereals from spanish market. Food Control 2011, 22: 1949-55.

48. Riba A, Bouras N, Mokrane S, Mathieu F, Lebrihi A, Sabaou N. Aspergillus section Flavi and aflatoxins in Algerian wheat and derived products. Food Chem Toxicol. 2010; 48: 2772-7.

49. Haidukowski M, Pascale M, Perrone G, Pancaldi D, Campagna $C$, Visconti $A$. Effect of fungicides on the development of Fusarium head blight, yield and deoxynivalenol accumulation in wheat inoculated under field conditions with Fusarium graminearum and Fusarium culmorum. J Sci Food Agr. 2005; 85: 191-8.

50. Stankovic S, Levic J, Ivanovic D, Krnjaja V, Stankovic G, Tancic S. Fumonisin $B 1$ and its co-occurrence with other fusariotoxins in naturally-contaminated wheat grain. Food Control 2012, 23: 384-8.

51. Kolmanic A, Simoncic A, Vajs S, Cencic A, Lesnik M. Fate of deoxynivalenol and nivalenol during storage of organic whole-grain wheat flour. J Stored Prod Res. 2010; 46: 66-71.

52. Tutelyan VA. Deoxynivalenol in cereals in Russia. Toxicology Letters 2004; 153: 173-9.

53. Del Ponte EM, Garda-Buffon J, Badiale-Furlong E. Deoxynivalenol and nivalenol in commercial wheat grain related to Fusarium head blight epidemics in southern Brazil. Food Chem. 2012; 132: 1087-91.

54. Pascale $M$, Panzarini $G$, Visconti A. Determination of HT-2 and $T-2$ toxins in oats and wheat by ultra-performance liquid chromatography with photodiode array detection. Talanta 2012; 89: 231-6.

55. Skrbic B, Zivancev J, Durisic-Mladenovic N, Godula M. Principal mycotoxins in wheat flour from the Serbian market: Levels and assessment of the exposure by wheat-based products. Food Control 2012; 25: 389-96.

56. Sulyok M, Berthiller F, Krska R, Schuhmacher R. Development and validation of a liquid chromatography/tandem mass spectrometric method for the determination of 39 mycotoxins in wheat and maize. Rapid Commun Mass Sp. 2006; 20: 2649-59.

57. Cervino C, Asam S, Knopp D, Rychlik M, Niessner R. Use of isotope-labeled aflatoxins for LC-MS/MS stable isotope dilution analysis of foods. J. Agric. Food Chem. 2008; 56: 1873-79.

58. Santos JS, Oliveira TM, Martins LM, Hashimoto EH, Bassói MC, Pires LF, Miranda MZ, Garcia S, Itano EN, Ono YS, Kawamura O, Hirooka EY. Monitoramento e nível de ingestão de desoxinivalenol por trigo. Semina Ciênc Agrar.2011; 32: 1439-50.

59. Ramos CR, Brasil EM, Geraldine RM. Avaliação de métodos de extração, limpeza e purificação de aflatoxinas para análise em cromatografia líquida de alta eficiência. Pesq Agropec Trop. 2008; 38: 103-8.
60. Hope R, Magan A. Comparison of environmental profiles for growth and deoxynivalenol production by Fusarium culmorum and F. graminearum on wheat grain. Lett Appl Microbiol. 2005, 40: 295-300.

61. Fujii S, Garcia LB, Hirooka EY. Metodologia analítica imunoquímica com ênfase na detecção de micotoxinas ficotoxinas no sistema agroalimentar. Alim Nutr. 2004; 15: $273-84$.

62. Odoemelam SA, Osu Cl. Aflatoxin B1 contamination of some edible grains marketed in Nigeria. E-J Chem. 2009; 6: 308-14.

63. Sahar N, Ahmed M, Parveen Z, Ilyas A, Bhutto A. Screening of mycotoxins in wheat, fruits and vegetables grown in Sindh, Pakistan. PakJ Bot. 2009; 41: 337-41.

64. Atalla MM, Hassanein NM, El-Beih AA, Youssef YAG. Mycotoxin production in wheat grains by different Aspergilli in relation to different relative humidities and storage periods. Nahrung/Food. 2003; 47: 6-10.

65. Saeger SD, Sibanda L, Paepens C, Lobeau M, Delmulle B, Barna-Vetro I, Peteghem CV. Novel developments in rapid mycotoxin detection. Mycotoxin Res. 2006; 22: 100-4.

66. Lupo A, Roebuck C, Settimo K, Quain A, Kennedy J, Abouzied $M$. Validation study of a rapid ELISA for detection of deoxynivalenol in wheat, barley, malted barley, corn, oats, and rice. J AOAC Int. 2010; 93: 600-10.

67. Garcia MJM, Biaggioni MAM, Ferreira WA, Kohara $E Y$, De Almeida AM. Sucessão de espécies de fungos em milho armazenado em sistema aerado. Rev Bras Armaz. 2003; 27: 14-22.

68. EC. European Commission. Commission Decision 2002/657/EC of 12 August 2002 implementing Council Directive 96/23/EC concerning the performance of analytical methods and the interpretation of results. Official J European Com. 2002b.

69. Bravin, F, Duca RC, Loiseau N, Pean M, Puel O, Delaforge $M$. Production and use of mycotoxins uniformly enriched with stable isotopes for their dosage in biological samples. World Mycotoxin J. 2008; 1: 275-81.

70. Birck NMM, Lorini I, Scussel VM. Fungus and mycotoxins in wheat grain at post harvest. 9th International Working Conference on Stored Product Protection. 2006; 198- 205.

71. WEB of Knowledge. Aflatoxin M1: Citations in Each Year. Disponible en: apps.isiknowledge.com. Consultado en Octubre de 2012.

72. Mallmann CA, Almeida CAA, Dilkin P, Sturza DAF, Vasconcelos TG, Rauber R. Determinação de desoxinivalenol em grãos e cereais por cromatografia líquida de alta eficiência com detecção por espectrometria de massa (LC/MS). Rev Cienc Vida 2008; 28: 79-81.

73. Terken B, Ayse BE, Gozde G, Sevtap A, Gonul S. Aflatoxin and ochratoxin in various types of commonly consumed retail ground samples in Ankara, Turkey. Ann Agric Environ Med. 2005; 12: 193-7. 